\title{
Fenofibrate-Associated Creatinine Increase: A Case for More Prospective Studies
}

\author{
Vusimuzu Ncube ${ }^{\mathrm{a}}$, Bryan Starkey ${ }^{\mathrm{b}}$, Tim Wang, ${ }^{\mathrm{a}, \mathrm{b}}$
}

\section{To the Editor}

We read with interest a recently published study by Abbas et al in which the authors discussed the factors associated with the well-established rise in creatinine associated with fibrate treatment. They retrospectively analysed the case notes from a lipid clinic database of 132 patients [1].

In accord with a number of studies published in the last 13 years (Tsimihodimos et al [2], Hottelart et al [3], Ansquer et al [4] ), interalia, the authors have shown a fibrate-associated increase in creatinine. In a recent prospective study, in hyperlipidaemic patients attending a lipid clinic [5] we have demonstrated a similar rise in serum creatinine using a Siemens enzymatic creatinine assay (Siemens Healthcare Diagnostics, Frimley, UK), which is traceable to isotope dilution/ mass spectrometry (ID/MS). This rise in creatinine (median 15.1\%; range 5.5-23.2 \%) was accompanied by an increase in cystatin C (median 9.9\%; range 1.1-26.1\%) in 10 of the 12 patients studied.

We concluded that since the increase in serum creatinine concentration was accompanied by a rise in cystatin $C$, an independent marker of GFR, the fibrate-related creatinine increase reflects a genuine reduction in renal function rather than an increased metabolic production of creatinine as previously proposed by Hottelart et al [3]. It is unlikely that another mechanism is responsible since it would need to affect both creatinine and cystatin $\mathrm{C}$ in a similar manner. Although our study was limited to only 12 patients, our findings reflect those of larger studies, for example, a substudy of the Fenofibrate Intervention and Event Lowering in Diabetes

\footnotetext{
Manuscript accepted for publication October 4, 2012

${ }^{\mathrm{a}}$ Frimley Park Hospital NHS Foundation Trust, UK

${ }^{\mathrm{b}}$ Royal Surrey County Hospital NHS Foundation Trust and Frimley Park Hospital NHS Foundation Trust, UK

${ }^{\mathrm{c}}$ Corresponding author: Tim Wang, Department of Clinical Biochemistry, Frimley Park Hospital NHS Foundation Trust, Portsmouth Road,

Frimley, Surrey, GU16 7UJ, UK. Email: tim.wang@fph-tr.nhs.uk
}

doi: http://dx.doi.org/10.4021/wjnu55w
(FIELD) trial [6].

Recently, Mychaleckj et al [7] have shown a reversal of the fibrate-induced rise in cystatin $\mathrm{C}$ concentration on stopping fibrate treatment, which further suggests that fibrateinduced renal hypofunction occurs.

In the study by Abbas et al [1] the use of a Jaffe creatinine assay presents a potential problem, since, unlike the enzymatic method used in our study, this method is prone to a range of analytical interferences. The findings, however, are still of interest. Although the dose of fenofibrate used was not stated, (and was probably variable), the authors have interestingly, corrected their data for the duration of treatment.

We speculate that some of the differences seen between studies may be related to fenofibrate dose in addition to the duration of therapy. There is a need for more prospective studies investigating the mechanism(s) and clinical significance of a fibrate-associated creatinine increase. The measurement of GFR using more accurate methods such as Inulin or para amino hippurate (PAH) clearance do not lend themselves to routine clinical use, but may currently offer the best prospect for gaining a clearer insight into this interesting phenomenon.

\section{References}

1. Abbas A, Sarafa S, Ramachandran S, Raju J, Ramachandran S. Factors Associated With Fibrate-Induced Creatinine Elevation: Observations in an Outpatients Setting. World J Nephrol Urol. 2012; 1(2-3):51-58

2. Tsimihodimos V, Kakafika A, Elisaf M. Fibrate treatment can increase serum creatinine levels. Nephrol Dial Transplant. 2001;16(6):1301.

3. Hottelart C, El Esper N, Rose F, Achard JM, Fournier A. Fenofibrate increases creatininemia by increasing metabolic production of creatinine. Nephron. 2002;92(3):536-541.

4. Ansquer JC, Dalton RN, Causse E, Crimet D, Le Malicot $\mathrm{K}$, Foucher C. Effect of fenofibrate on kidney function: a 6-week randomized crossover trial in healthy people. Am J Kidney Dis. 2008;51(6):904-913.

5. Ncube V, Starkey B, Wang T. Effect of fenofibrate treat- 
ment for hyperlipidaemia on serum creatinine and cystatin C. Ann Clin Biochem. 2012;49(5):491-493.

6. Forsblom C, Hiukka A, Leinonen ES, Sundvall J, Groop PH, Taskinen MR. Effects of long-term fenofibrate treatment on markers of renal function in type 2 diabetes: the FIELD Helsinki substudy. Diabetes Care.
2010;33(2):215-220.

7. Mychaleckyj JC, Craven T, Nayak U, Buse J, Crouse JR, Elam M, Kirchner K, et al. Reversibility of fenofibrate therapy-induced renal function impairment in ACCORD type 2 diabetic participants. Diabetes Care. 2012;35(5):1008-1014. 